\title{
ACTUALIDAD POLÍTICA Y CONSTITUCIONAL FRANCESA (2005-2006)
}

\author{
ÁNGEL J. SÁNCHEZ NAVARRO \\ Profesor Titular de Derecho Constitucional \\ Universidad Complutense de Madrid
}

\author{
Sumario \\ I. Introducción. \\ II. Hacia las presidenciales de 2007: Ajustes \\ legislativos. \\ III. La polémica reforma laboral. \\ IV. Otras disposiciones de interés.
}

\section{INTRODUCCIÓN}

Cualquier seguidor no ya de estas "crónicas" que Teoría y Realidad Constitucional viene publicando desde su nacimiento, sino de la vida política y constitucional francesa que reflejan los medios de comunicación españoles puede apreciar la situación de relativo desconcierto que atraviesa nuestro país vecino. Un desconcierto que no sólo protagoniza algunos polémicos ensayos de notable difusión ${ }^{1}$, sino que se ha visto, simultáneamente, revelado y reforzado por acontecimientos muy diversos. Algunos de ellos han sido recogidos ya en estas páginas (en particular, los resultados de la primera vuelta de las elecciones presidenciales de la primavera de 2002 y la respuesta negativa en el referéndum sobre el Tratado por el que se establece una Constitución para Europa $)^{2}$, pero no son ni mucho menos los únicos. Sin ir más lejos, en los últimos meses se han producido otras situaciones de crisis (plasmadas, en primera instancia, en revueltas callejeras, y que encontraron eco posteriormente, como tendremos ocasión de ver, en disposiciones de naturaleza legislativa) como las

1 Véase, entre los más conocidos, el conocido libro de Nicolas BAVEREZ, La France qui tombe (Paris, Perrin, 2003; versión española: Francia en declive, Madrid, Gota a gota, 2005).

2 Vide TRC, números 10-11 (pp. 549-556), 15 (passim) y 16 (pp. 397-399). 
que, en la primera mitad de noviembre de 2005, se extendieron desde la periferia de París a todas las grandes ciudades galas; o las que, en marzo y abril de este mismo año, acabaron por desfigurar la reforma laboral inicialmente propuesta por el Gobierno.

En ese contexto, resulta lógico constatar la ausencia de reformas constitucionales, y la relativa escasez de disposiciones legislativas de relieve constitucional. Leyes que, por lo demás, obedecen en buena parte a la necesidad de adecuar el marco institucional a las nuevas condiciones derivadas del mandato presidencial, recortado a cinco años por la Ley Constitucional 2000-964, de 2 de octubre $^{3}$. En consecuencia, en las páginas siguientes examinaremos, en primer lugar, este conjunto de ajustes legislativos orientados a las elecciones presidenciales (II); a continuación, trataremos la polémica suscitada por la reforma laboral y, en particular, por la figura del "Contrato de Primer Empleo" (CPE) (III); para acabar con una mención, siquiera mínima, a otras disposiciones de interés (IV).

\section{HACIA LAS PRESIDENCIALES DE 2007: AJUSTES LEGISLATIVOS}

Como ya se apuntó al exponer la reforma constitucional del año 2000, el recorte del mandato presidencial y la subsiguiente coincidencia de las elecciones presidenciales y legislativas en un mismo año redujeron considerablemente las posibilidades de cohabitación entre mayorías distintas y, como lógica consecuencia, de disolución anticipada de la Asamblea Nacional. La confirmación de esas expectativas supone que en 2007 habrá de repetirse la experiencia de la doble cita electoral de 2002. En definitiva, otra vez los partidos se ven sometidos a un desafío en el que lo normal es que los resultados de las presidenciales se reproduzcan, poco más tarde, en las legislativas. Lo que está en juego es, si cabe, mucho más que antes, y ello explica que la vida política e institucional esté casi exclusivamente marcada por el horizonte electoral. Tanto más cuando las circunstancias favorecen la incertidumbre no ya en cuanto al resultado, sino incluso en cuanto a la identidad de los posibles contendientes.

En efecto, tras doce años de mandato, no parece probable que el Presidente Chirac repita como candidato. Ello, unido a la inhabilitación penal de quien parecía llamado a ser su "delfín", Alain Juppé, ha desencadenado una pugna dentro del partido mayoritario, la Union pour un Mouvement Populaire (UMP), que actualmente parece decantarse a favor de su Presidente (y actual Ministro del Interior), Nicolas Sarkozy, aunque no sea posible excluir una posible candidatura "potente" del Primer Ministro Dominique De Villepin, hombre de absoluta confianza de Chirac. Mientras que, en la izquierda, las cosas tampoco están claras: aunque parezca poco probable, como mínimo, que un Laurent Fabius reforzado por su oposición al Tratado Constitucional Europeo sea respaldado por el Partido Socialista, lo cierto es que el líder y Primer Secretario 
del mismo, François Hollande, presenta un perfil bajo que ha favorecido la aparición de nuevos "precandidatos". Entre ellos, destaca especialmente la ex -ministra y actual Presidenta socialista de la región Poitou-Charentes, Ségoléne Royal (compañera sentimental del propio Hollande), sin descartar a otros como Lionel Jospin, quien se ha mostrado "disponible".

Añádase a ello la presencia, segura, probable o, simplemente, posible, de otros partidos y candidatos menores, pero significativos. Así, en el campo del centro-derecha, parecen seguras al menos las candidaturas de la UDF y el soberanista MPF (con los tenaces François Bayrou y Philippe De Villiers); mientras que, en el resto de la izquierda, están confirmadas - por ahora- las candidaturas de Los Verdes (Dominique Voynet), de los trotskistas (Besancenot y Laguiller), a las que podrían unirse las del PCF, el MRC de Chevènement $\mathrm{O}$, incluso, el líder sindical agrario, José Bové... Todo lo cual no permite descartar de plano la posibilidad de que se repita el shock de 2002, con el Front National de un Jean Marie Le Pen que usualmente obtiene en estos comicios sus mejores resultados.

Esta posibilidad ha encontrado, de hecho, algún eco doctrinal. En este sentido, dos prestigiosos constitucionalistas como Guy Carcassone y Olivier Duhamel proponían recientemente, en un artículo periodístico ${ }^{4}$, ni más ni menos que modificar el sistema que rige el momento central de la vida política francesa bajo la V República, como es el de la elección presidencial. Alegando que "Francia no puede jugarse su futuro a la ruleta", estos autores partían del dato de que "un número elevado de candidatos incrementa considerablemente, y en proporciones considerables, el riesgo de una segunda vuelta no deseada, es decir una segunda vuelta en la que figure un candidato que ya ha reunido la totalidad de sus votos, mientras que queda eliminado otro que podría reunir muchos más sufragios". Es más: llegan a plantear situaciones potencialmente aún más perturbadoras que la de 2002, como que «los dos primeros candidatos sean los de formaciones extremistas que han presentado un frente unido de cada lado, mientras que las grandes coaliciones han presentado varios candidatos cada una"; o que "el candidato de un extremo sea el más votado, mientras que los dos siguientes no están separados más que por un puñado de sufragios, hasta el punto de que el futuro vencedor habrá sido designado, de hecho desde la primera vuelta, y más por accidente que por voluntad de las urnas".

Ante tal panorama, Carcassone y Duhamel descartan la posibilidad —en principio, mucho más sencilla - de restringir las candidaturas presidenciales (que requieren, como es bien sabido, la firma de un cierto número de cargos públicos, que sería posible incrementar). A su juicio, ello provocaría numerosas protestas "que irían mucho más allá de los extremos, en tanto los diferentes movimientos políticos tienen las presidenciales como el momento privilegiado, si no único, de expresión política verdaderamente pluralista". Y, en consecuencia, optan por introducir la posibilidad de una vuelta intermedia si no llegan a dos

4 "Eviter un nouveau 21 avril" (Le Monde, 7 de marzo de 2006). 
los candidatos que reúnen el $20 \%$ de los sufragios emitidos en la primera vuelta. Si más de dos candidatos han superado ese umbral, nada cambia, y los dos que han reunido más votos se enfrentan normalmente en la segunda vuelta. Si menos de dos candidatos han alcanzado ese umbral, se organizaría una vuelta intermedia, el domingo siguiente, en la cual participarían los cuatro candidatos que han obtenido más votos en la primera. Sólo los dos primeros en esta segunda vuelta participarían en la votación decisiva, quince días más tarde».

Se trata, como es obvio, de una propuesta compleja, estrictamente calificable de ejemplo de ingeniería constitucional, con pocas posibilidades reales de ser aceptada. Incluso los sectores más detractores del sistema político vigente en Francia, que consideran que los últimos resultados de las presidenciales y del referéndum europeo ponen de manifiesto el alejamiento entre los ciudadanos y las instituciones, estiman que no resuelve nada, y no es sino un intento de "recomponer" un régimen político que ya no funciona adecuadamente. En otro orden de cosas, y con carácter más de curiosidad que de argumento útil para este posible debate, cabe recordar que tampoco puede considerarse que esta fórmula sea especialmente novedosa: en efecto, el Reglamento seguido para la formación de los État Généraux de 1789 establecía, en su artículo 47, que la elección de diputados tenía lugar mediante un sistema uninominal, mayoritario y a tres vueltas, exigiéndose mayoría absoluta de los votos en las dos primeras y limitándose la participación en la última de ellas tan sólo a "los dos sujetos que han reunido más votos" ${ }^{5}$.

De cualquier modo, y al margen de estas disquisiciones doctrinales, lo cierto es que la adopción del mandato de cinco años para el Presidente de la República ha obligado a hacer otras adaptaciones. Por expresarlo en los prolijos, pero expresivos, términos utilizados por la "Exposición de motivos" del proyecto de ley que ha permitido prorrogar el mandato de los consejeros municipales y de los consejeros generales renovables en 2007, «el calendario electoral de 2007 presenta[ba] dificultades importantes de puesta en práctica. En el curso de este año deben renovarse los mandatos del Presidente de la República, elegido en 2002; de los diputados elegidos el mismo año; de los consejeros generales y municipales elegidos en 2001 y de los senadores electos en 1998 ".

Así las cosas, «el espíritu de las instituciones de la V República y la inversión del calendario electoral por la Ley orgánica... que modificó la fecha de expiración de los poderes de la Asamblea Nacional hacen inoportuna cualquier modificación de la secuencia de los meses de abril a junio de 2007 en el curso de la cual se suceden el escrutinio presidencial y el legislativo. Esta secuencia resulta de las fechas de expiración de los poderes del Presidente de la Repú-

5 Règlement électoral de 1789 intitulé "Règlement fait par le Roi pour l'execution des lettres de convocation", en J.B. DUVERGIER, "Collection complète des Lois, Décrets, Réglemen[t]s, avis du Conseil d'État (de 1789 à 1830)", Paris, A. Guyot, 1834, vol. I; reproducido por J. CADART, Le régime électoral des États Généraux de 1789 et ses origines (1302-1614), Annales de l'Université de Lyon, Fascicule n. 11, Paris, Sirey, 1952, pp. 192-201). 
blica y de los diputados, fijadas respectivamente para el 17 de mayo y el 19 de junio de $2007^{6}$.

Por el contrario, la renovación de los consejos municipales no puede tener lugar en las condiciones fijadas por el artículo 227 del Código Electoral, esto es, en el mes de marzo del sexto año que sigue a su elección. En efecto, la organización de tales comicios en las semanas que preceden a la elección presidencial no permitiría a los candidatos a la Presidencia de la República recoger de manera serena y equitativa los avales [parrainages] de quinientos cargos electos..., como les exige la Ley orgánica... relativa a la elección del Presidente de la República por sufragio universal. A ello se añaden las dificultades que encontrará la Comisión Nacional de Cuentas de Campaña y de Financiación Política para el tratamiento de las cuentas de los candidatos en los plazos establecidos por la legislación.

La fecha de renovación de los consejeros municipales electos en 2001 debe ser, pues, modificada. Y a fin de mantener la concomitancia de los comicios locales, la fecha de renovación de los consejeros generales electos en 2001 queda fijada el mismo día fijado para los municipales.

El hábito republicano se opone a que los mandatos de los consejeros municipales y generales sean recortados por una ley posterior a las elecciones correspondientes. Las elecciones municipales y cantonales deben así posponerse a las presidenciales y legislativas de la primavera de 2007.

Estas elecciones no pueden tener lugar al mismo tiempo que la renovación de los senadores elegidos en 1998, normalmente prevista para septiembre de 2007. Las elecciones senatoriales deben igualmente trasladarse. En efecto, los consejeros municipales y generales representan la mayor parte de los grandes electores del Senado. Parece pues legítimo que la renovación de los consejos municipales y generales preceda al de esta Cámara”.

Así las cosas, "Las elecciones municipales y cantonales se producirán en marzo de 2008", y los mandatos de los consejeros así electos expirarán "en marzo de 2014, conforme a las disposiciones de derecho común en vigor. Por el contrario, la preservación del ritmo resultante de la renovación trienal por mitades de los consejos generales obliga a retrasar igualmente en un año - esto es, hasta marzo de 2011 - la renovación de los consejeros generales elegidos el 21 y 28 de marzo de 2004. De no hacerse así, el retraso desde 2007 a 2008 de las elecciones cantonales modificaría profundamente el modo de funcionamiento de los consejos generales, ya que las fechas de renovación siguientes estarían espaciadas alternativamente de cuatro y de dos años, con elecciones en 2008, 2010, 2014, 2016, 2020, etc. La duración del mandato de los ejecutivos departamentales sería entonces fijada alternativamente en dos y cuatro años, lo que constituiría una fuente de perturbación del funcionamiento de los departamentos".

En definitiva, el horizonte electoral de la primavera de 2007 ha exigido aprobar normas diversas y de distinto rango, por imperativo constitucional. En

6 Sobre esta Ley orgánica, vide TRC, n. ${ }^{\circ} 7$, pp. 288-290. 
efecto, es preciso considerar que la Constitución reserva a la ley orgánica la regulación tanto de la elección del Presidente de la República como de ciertos aspectos del régimen jurídico de diputados y senadores ${ }^{7}$. Mientras que deja en manos del legislador ordinario, en particular, «las reglas concernientes al régimen electoral de las asambleas parlamentarias y... territoriales" (artículo 34). De esta manera, deben mencionarse:

\section{A) La Ley orgánica Relativa a la elección del Presidente de la República}

Desde hace ya varias décadas, la práctica institucional francesa ha adoptado la costumbre de aprobar, de acuerdo con la ya mencionada reserva del artículo 6 de la Constitución, una Ley orgánica específica previa a cada elección presidencial ${ }^{8}$. Una Ley orgánica que, según otra tradición republicana, no debe afectar esencialmente a la igualdad entre los potenciales candidatos, lo que evidentemente limita su alcance, y que en la actualidad se tramita antes de que se inicie el período preelectoral de un año en el que se aplica la Ley orgánica de financiación electoral. En definitiva, esta Ley permite, de una parte, aplicar a la elección presidencial aquellos aspectos del régimen electoral ordinario que han sufrido modificaciones (aprobadas por leyes ordinarias) en los años anteriores; y, de otra, introducir las innovaciones que se estimen oportunas, y que muy especialmente atienden a las observaciones formuladas, tras cada proceso electoral, por el Consejo Constitucional, a quien corresponde "velar por la regularidad de la elección del Presidente de la Repúblican, examinando las reclamaciones y proclamando sus resultados (artículo $58 \mathrm{C}$.).

De esta manera, el Journal Officiel publicó el día 6 de abril la Ley orgánica relativa a la elección del Presidente de la República9. Una Ley que no contó con apoyo unánime: el Partido Socialista afirmó su acuerdo sustancial con un contenido "que aporta mejoras técnicas y simplifica diversos procedimientos". Ahora bien, dado que, a su juicio, el texto debía incorporar algunas precisiones relativas a aspectos sustanciales (como el estatuto penal y económico del Presidente de la República); y que, por otra parte, no se habían atendido algunas de sus enmiendas más "técnicas", optó por la abstención. Mientras que los Grupos Comunistas de ambas Cámaras consideraban que «la crisis social, económica e institucional" francesa merecía un "debate de fondo sobre las instituciones", que en particular "redujese fuertemente el papel del Presidente de la República", restableciendo "la preponderancia del legislativo sobre el ejecutivo" a fin de "devolver el poder a los ciudadanos". Como resulta obvio que esta Ley

7 «El Presidente de la República es elegido por cinco años mediante sufragio universal directo. Una Ley orgánica fijará las modalidades de aplicación del presente artículo" (artículo 6, reformado en el año 2000); y “Una Ley orgánica fijará la duración de los poderes de cada Cámara, el número de sus miembros, su retribución, las condiciones de elegibilidad..." (artículo 25).

8 Así ocurrió, por ejemplo, en 1988, 1995 y 2001.

9 Loi organique n. 2006-404, du 5 avril 2006. 
orgánica no satisfacía tales posiciones, cuyo objetivo último era el de "una VI República, que se inscribiría en la continuidad de una tradición democrática nacida en 1789 , pero que al mismo tiempo sería el principio de una nueva era democrática", estos Grupos optaron por no participar en la votación (en la Asamblea Nacional) o por votar en contra (en el Senado) ${ }^{10}$.

En cuanto a su contenido, esta Ley, declarada conforme tras el preceptivo examen del Consejo Constitucional ${ }^{11}$, modifica esencialmente (aunque no sólo) algunos aspectos de la Ley orgánica 62-1292, de 6 de noviembre de 1962, que regula la elección del Presidente de la República. Sus puntos principales son los siguientes:

a) En primer lugar, y conforme a las sugerencias elaboradas por el propio Consejo tras la última elección de 2002, se adelanta el plazo para la presentación de los avales exigidos para ser candidato (un mínimo de $500^{12}$ ). Concretamente, si hasta el momento los avales debían presentarse "al menos dieciocho días antes de la primera vuelta", la nueva ley obliga a hacerlo "antes de las 18 horas del sexto viernes anterior" a dicha primera votación (es decir, 37 días). Las razones que justifican este cambio son diversas, destacándose que otorga al Consejo Constitucional un mayor plazo - el doble de tiempo- para poder verificar la validez de los avales, permitiéndole además hacer pública antes la relación definitiva de candidatos, lo cual reduce el período de incertidumbre en el que los ciudadanos no conocen qué "precandidatos" llegarán a reunir los avales requeridos.

b) En segundo término, y siguiendo así mismo otra sugerencia del Consejo Constitucional, el control de las cuentas de los candidatos a la Presidencia, hasta la fecha atribuida al propio Consejo, se transfiere a la "Comisión Nacional de Cuentas de Campaña y de Financiación Política", cuyas decisiones podrán recurrirse ante el mismo Consejo. De esta manera, dicha Comisión (configurada como «autoridad administrativa independiente" desde noviembre de 2003) completa su función controladora, que venía ejerciendo ya en todas los demás consultas electorales salvo en las presidenciales. Las ventajas derivadas de esta innovación son obvias, destacando la seguridad jurídica derivada de la

10 Las referencias entrecomilladas proceden de las intervenciones de los diputados R. Dosière (PS) y M. Vaxès (PC), y de la senadora N. Borvo Cohen-Seat (PC), en los debates celebrados en la Asamblea Nacional y en el Senado, los días 21 y 29 de marzo, respectivamente.

11 Decisión n. 2006-536 DC du 5 avril 2006: recuérdese que, según los artículos 46 y 61 de la Constitución, las leyes orgánicas sólo pueden promulgarse tras la declaración de su conformidad a la Constitución por parte del Consejo.

12 Durante el debate de la Ley se ha apuntado, en diversas ocasiones, la conveniencia de incrementar este número de "padrinazgos" (parrainages) que, como se ha apuntado, debe proceder de cargos electos, a fin de restringir el número de candidatos presidenciales. Sin embargo, el alcance limitado de esta norma, y la proximidad del período electoral, llevaron a descartar esta discusión, que posiblemente pueda replantearse en el futuro. 
existencia de un único órgano especializado, encargado de cumplir esta función en todo tipo de procesos electorales, armonizando pues los criterios aplicados; la garantía que supone la configuración de una doble instancia antes inexistente; la liberación de tiempo y esfuerzo que ello supone para el Consejo Constitucional, etc.

c) En tercer lugar, se refuerza el margen de apreciación tanto de la citada Comisión Nacional como del Consejo Constitucional a la hora de determinar la sanción aplicable en caso de que se aprecie alguna irregularidad en las cuentas de un candidato. Frente a la situación anterior, en la cual el Consejo Constitucional no tenía otra opción más que la de otorgar o negar completamente la subvención correspondiente, el artículo 4 de la Ley dispone que "en los casos en que las irregularidades cometidas no conduzcan al rechazo de las cuentas, la decisión.. puede reducir la cuantía de la subvención electoral [remboursement forfaitaire] en función del número y la gravedad de tales irregularidades".

d) Una cuarta innovación, cuya significación política va probablemente mucho más allá de la que parece desprenderse a primera vista, es la que extiende a las elecciones presidenciales el principio de la votación en sábado para ciertos lugares, ya introducido en anteriores consultas (europeas de 2004 y referéndum de mayo de 2005). La reforma responde al hecho constatable de que, por la diferencia horaria, los ciudadanos franceses residentes en los Departamentos y territorios de Ultramar y en territorio americano emitían su sufragio cuando ya se conocían los resultados globales de la metrópoli, reduciendo así la significación del mismo. Para evitarlo, el Consejo Constitucional había propuesto que en tales territorios la elección se produjese el sábado anterior. Esta modificación se facilitó con la ley constitucional de 2003, que alteró muy levemente el artículo 7, el cual disponía hasta entonces que la segunda vuelta tendría lugar, en su caso, "el segundo domingo siguiente" a la primera. Pues bien, desde 2003 esa referencia fue reemplazada por otra que aludía "al decimocuarto día siguiente, ${ }^{13}$. Desaparecida la mención al domingo, la Ley orgánica dispone en su artículo 2.4 que "por derogación de las disposiciones del artículo 55 del Código Electoral, la votación se organizará el sábado en Guadalupe, Guayana, Martinica, Saint-Pierre-et-Miquelon, en la Polinesia francesa y en las embajadas y puestos consulares situados en el continente americano".

e) En otro orden de cosas, la Ley orgánica dispone que todas las remisiones a otras normas (principalmente, al Código Electoral) que en ella se contienen se entienden referidas a la redacción que tales normas tienen en la fecha de aprobación de la misma, esto es, el 5 de abril de 2006. En consecuencia, cualquier reforma posterior en dichas normas no será

13 TRC, núm. 12-13 (2004), pp. 486 y ss., especialmente 491. 
aplicable a sucesivas elecciones presidenciales salvo que la correspondiente ley orgánica así lo disponga expresamente.

f) Finalmente, la Ley modifica también un apartado de otra Ley orgánica, la que regula la participación de los electores franceses residentes fuera de Francia en la elección presidencial, a la que volveremos inmediatamente ${ }^{14}$. Concretamente, su artículo 4.2 permite que las listas electorales consulares incluyan la dirección electrónica de cada elector, a fin de facilitar la difusión de la información relativa a la elección. Una disposición introducida por la ponencia de la Asamblea Nacional, y que sin duda puede ser útil a fin de agilizar la circulación de la información electoral.

\section{B) LA LEY ORGÁNICA RELATIVA A LA PARTICIPACIÓN EN LAS ELECCIONES PRESIDENCIALES DE LOS FRANCESES RESIDENTES EN EL EXTRANJERO}

Pocos meses antes, en julio de 2005, y siempre respetando la mencionada reserva constitucional, otra Ley orgánica reformó la anteriormente citada Ley orgánica reguladora de la participación de los electores franceses residentes fuera de Francia en la elección presidencial ${ }^{15}$. Esta Ley se limita básicamente a armonizar y simplificar las condiciones de voto de los franceses residentes en el extranjero en las elecciones presidenciales, por lo que no encontró ninguna oposición parlamentaria y fue declarada conforme a la Constitución por el Consejo Constitucional ${ }^{16}$.

En cuanto a su contenido, cabe destacar en particular que la Ley unifica el censo de electores franceses residentes en el extranjero, y facilita el voto por procuración, disponiendo en su artículo 13 que "los electores inscritos en una lista electoral consular pueden ejercer, a solicitud propia, su derecho de voto por procuración cuando certifiquen por su honor [lorsqu'il attestent sur l'bonneur] que les resulta imposible personarse en la mesa el día de la votación". De esta forma, se facilita el voto de muchos franceses que residen a decenas, e incluso centenares de kilómetros, de las dependencias diplomáticas donde deberían ejercer su sufragio ${ }^{17}$.

14 Loi organique n. ${ }^{\circ}$ 76-97, du 31 janvier 1976, relative aux listes électorales consulaires et au vote des Français établis hors de France.

15 Loi organique n. ${ }^{\circ}$ 2005-821, du 20 juillet 2005 modifiant la loi organique n. $76-97$ du 31 janvier 1976 sur le vote des Français établis hors de France pour l'élection du Président de la République.

16 Décision n. 2005-518 DC du 13 juillet 2005.

17 Aun cuando no tenga relación con la elección del Presidente de la República, debe mencionarse que en la misma fecha de 20 de julio se aprobó otra Ley $\left(n .^{\circ}\right.$ 2005-822) que modificó la ley n. ${ }^{\circ}$ 82-471, de 7 de julio de 1982, relativa a la Asamblea de los Franceses del Extranjero (AFE). En efecto, hasta este momento la elección de esta Asamblea utilizaba un censo electoral específico, que desaparece ante la apuntada creación de un "censo unificado" de franceses residentes en el extranjero. 


\section{C) La Ley orgánica que modifica las fechas de Renovación del Senado}

Una tercera Ley orgánica es la que, de acuerdo con las razones ya expuestas, ha debido modificar las fechas de renovación de los escaños del Senado ${ }^{18}$. Porque, en definitiva, el problema suscitado por la acumulación de citas electorales en 2007 se proyecta hacia el futuro, de modo que el retraso hasta 2008 en la elección de los senadores que debían renovarse en 2007 se amplía a todos los demás: el mandato de los senadores renovables en septiembre de 2010 se prorroga hasta septiembre de 2011, y lo mismo ocurre con la renovación de los escaños que debía tener lugar en septiembre de 2013, que se pospone hasta el mismo mes del año siguiente. En consecuencia, se reforma "a título transitorio" el artículo LO 275 del Código Electoral, así como la Ley orgánica de 30 de julio de 2003 que preveía la renovación por mitades del Senado y la ampliación del número de sus miembros ${ }^{19}$.

Una vez más, y como ya ocurriera con la Ley orgánica que prorrogó en 2001 el mandato de la Asamblea Nacional a fin de posponer su elección a las presidenciales de 2002, el Consejo Constitucional hubo de pronunciarse sobre la adecuación constitucional de esta disposición. Y lo hizo en términos similares a los utilizados en aquella ocasión, considerando que "el legislador orgánico, competente... para fijar la duración de los poderes de cada Asamblea, puede modificar[la] por un fin de interés general y... con respeto a las reglas y principios de valor constitucional; que el Consejo Constitucional no dispone de un poder general de apreciación y de decisión de la misma naturaleza que el del Parlamento; que, en consecuencia, no le corresponde investigar si el objetivo perseguido por el legislador podría alcanzarse por otras vías, en tanto las modalidades adoptadas por la ley no sean manifiestamente inapropiadas para tal fin". Así las cosas, tras resaltar que "la prórroga de los mandatos senatoriales reviste un carácter excepcional y transitorio", el Consejo consideró que la opción del legislador "no es manifiestamente inapropiada para el objetivo perseguido", por lo que declaró que esta Ley orgánica no es contraria a la Constitución ${ }^{20}$.

\section{D) La Ley QUe PRORROGa la DURACIÓN DEL MANDATO DE LOS CONSEJEROS MUNICIPALES Y DE LOS CONSEJEROS GENERALES RENOVABLES EN 2007}

Por último, y fuera ya del ámbito constitucionalmente reservado a la Ley orgánica, ya se ha hecho anteriormente referencia a esta disposición, cuya Exposición de motivos explica claramente la necesidad de todas estas alteraciones en el calendario electoral galo. En definitiva, la ley (que, como la anterior, no

18 Loi organique n. 2005-1562 du 15 décembre 2005 modifiant les dates des renouvellements du Sénat.

19 Vide TRC, núm. 14 (2004), pp. 417-418.

20 Décision n. 2005-529 DC du 15 décembre 2005. 
obtuvo el apoyo de la oposición de izquierdas) modificó los artículos correspondientes del Código Electoral y de la ley ordinaria que reformó el procedimiento de elección de los senadores, posponiendo a 2008 y 2011 los procesos electorales mencionados que habían de tener lugar en 2007 y $2010^{21}$.

\section{LA POLÉMICA REFORMA LABORAL}

Apuntábamos ya en la introducción a estas páginas el que, sin duda, ha sido uno de los conflictos sociales más relevantes acaecidos en los últimos meses, y no sólo en Francia. Como es sabido, la polémica se suscitó en torno a, y acompañó a toda la tramitación de, un proyecto de ley "para la igualdad de oportunidades", que finalmente fue aprobado con el apoyo casi exclusivo del partido mayoritario, la $\mathrm{UMP}^{22}$.

Esta Ley encuentra su origen en un proyecto de ley aprobado por el Consejo de Ministros del 11 de enero de este mismo año, y constituye un ejemplo más de una ley relativamente larga, compleja y que afecta a diversas esferas. Concretamente, los 52 artículos de la Ley finalmente aprobada modifican diversas disposiciones de varios Códigos (de la Educación, del Trabajo, del Tributario, de la Seguridad Social, del Servicio Nacional, de la Sanidad Pública, de Comercio, de la Acción Social y de las Familias, de Procedimiento Penal); y de otras leyes, como las de ordenación del territorio, la de creación de una Alta Autoridad de lucha contra las discriminaciones y por la igualdad, la de libertad de comunicación... Todo lo cual se explica por unos objetivos ambiciosos, pero que afectaban a ámbitos muy distintos.

Así, entre sus objetivos declarados (que, como puede apreciarse a primera vista, en gran parte tratan de dar respuesta a la situación que revelaron las revueltas de octubre-noviembre de 2005) se encontraban, según la nota distribuida por el Gobierno al aprobar este proyecto, los de afavorecer el empleo de los jóvenes residentes en las zonas urbanas sensibles o con dificultades de inserción social y profesionalw; los de reforzar la igualdad de oportunidades mediante la creación de una "Agencia Nacional para la Cohesión Social y la Igualdad de oportunidades, a fin de incrementar la presencia del Estado en los barrios sensibles... y reforzar las acciones de integración". También se pretendía "ayudar a los padres a ejercer su autoridad parental", para lo cual se "instituye un contrato de responsabilidad parental, confiado a los consejos generales en relación con los servicios académicos", y que permitirá «asignar objetivos a los padres", abriendo la puerta a la posibilidad de suspensión provisional de las ayudas percibidas por las familias en caso de tales objetivos sean incumplidos.

21 Loi n. $^{\circ}$ 2005-1563 du 15 décembre.

22 La Loi n. $2006-396$ du 31 mars 2006 pour l'égalité des chances fue aprobada en la Asamblea Nacional el día 8 de marzo, por 329 votos a favor (328 UMP y 1 UDF) y 159 en contra (122 PSF, 14 UDF, 17 PC y PRG, 5 NI, 1 UMP), registrándose 4 abstenciones (1 UMP, 3 UDF). En el Senado, el 9 de marzo, los votos favorables fueron 178, y los contrarios 127, con 23 abstenciones. 
Además, reforzaba las facultades de los alcaldes ante la comisión de ciertos actos incívicos (incivilités), permitiéndoles proponer a la fiscalía algunas alternativas "sociales"; y, finalmente, pretendía "crear un servicio civil voluntario", que permitirá a los jóvenes adquirir una formación cívica y profesional.

Pese a todo ello, el debate se centró en las medidas de reforma laboral, y muy especialmente en el "Contrato de Primer Empleo" (CPE) previsto en el artículo 8 de la ley ${ }^{23}$. De acuerdo con este precepto, las empresas con más de veinte trabajadores pueden acudir a dicha figura contractual para emplear a jóvenes menores de 26 años. Ahora bien, lo que centró la polémica es que este tipo de contrato, que se establece por escrito y sin determinación de tiempo, permite a cualquiera de las partes, y evidentemente sobre todo al empleador, dejarlo sin efecto sin necesidad de motivar el despido durante un período de "Consolidación", de dos años de duración. Todo lo cual fue lógicamente objeto de una dura crítica por parte no sólo de la izquierda y de otros sectores políticos, sino también de los estudiantes, los sindicatos y otros grupos sociales (incluyendo a buena parte del empresariado), que consideraban principalmente que la reforma favorecería los despidos abusivos y la precariedad laboral.

La cuestión se vio además agravada por razones procedimentales: de una parte, la figura concreta del CPE fue introducida en la Asamblea Nacional por vía de enmienda, por lo que no fue examinada por el Consejo de Estado en su preceptivo dictamen sobre los proyectos de ley (artículo 39 C.); de otra, el Primer Ministro De Villepin planteó la responsabilidad de su Gobierno sobre el conjunto de la ley, según prevé el artículo 49.3 de la Constitución, lo que hizo que ésta fuese inicialmente aprobada sin votación en la Asamblea Nacional ${ }^{24}$.

Todas estas circunstancias justificaron no sólo la agria disputa política y social, sino también un intenso debate jurídico que, como es habitual, desembocó en un recurso planteado ante el Consejo Constitucional, el 14 de marzo, por diputados y senadores de la izquierda.

Como también es norma en la práctica institucional francesa, el debate jurídico-constitucional se zanjó con rapidez. Concretamente, el Consejo tardó apenas dos semanas en dictar su decisión ${ }^{25}$, que declaró conforme a la Constitución la mayor parte de la ley $-\mathrm{y}$, en particular, lo relativo al CPE-, declarando inconstitucionales dos artículos de la misma.

De esta forma, los artículos 21 y 22 de la ley no pudieron entrar en vigor por razones estrictamente procedimentales: según los considerandos 29 a 33 de la Decisión, ambos preceptos (que modificaban aspectos concretos del Código del Trabajo y de la ley de Presupuestos de la Seguridad Social para 2006) habían

23 En rigor, el Contrat Première Embauche no alude al primer empleo de un trabajador, sino a la primera contratación por una empresa de un trabajador, con independencia de que éste haya gozado antes o no de otro puesto de trabajo.

24 Añádase a ello que otra figura similar, el Contrat Nouvelle Embauche (CNE), destinada a las empresas con menos de veinte trabajadores, fue adoptada mediante ordenanza por el Gobierno De Villepin en agosto de 2005, fecha que evidentemente dificultaba cualquier reacción social. La medida, recurrida ante el Consejo de Estado, fue confirmada por éste.

25 Décision n. 2006-535 DC du 30 mars 2006 (J.O. del 2 de abril). 
sido introducidos por vía de enmienda, a pesar de que "están desprovistos de cualquier relación con el objeto del texto" sometido inicialmente a debate. Por tanto, esa falta de relación material con la "Ley para la igualdad de oportunidades" constituye, conforme a la reiterada doctrina del Consejo, una irregularidad de procedimiento que vicia estos preceptos, que son en consecuencia declarados contrarios a la Constitución sin necesidad de examinar ninguna otra alegación sobre los mismos.

Sin embargo, el mismo argumento es rechazado cuando se plantea respecto al artículo 8. Porque, como señalan los considerandos 4 a 11, el derecho de enmienda no tiene otros límites que los establecidos por las normas constitucionales, así como por la necesaria relación con el objeto del texto. De esta forma, y dado que el contenido del precepto sí guarda esa relación sustancial, la utilización - aun cuando sea cumulativa - de diversos mecanismos parlamentarios previstos en el ordenamiento francés no afecta a la constitucionalidad del procedimiento legislativo, que por lo tanto no puede considerarse como irregular.

De acuerdo con el contexto descrito, el examen del Consejo Constitucional se centra, sobre todo, en el reiterado artículo 8 (Considerandos 2 a 28 , sobre un total de 45). De este modo, y al margen del señalado debate procedimental, el Consejo descarta las alegaciones de que el precepto vulnera las exigencias de claridad e inteligibilidad de la ley (Considerandos 12 a 15), el principio de igualdad ante la ley ("ningún principio ni regla de valor constitucional prohíben al legislador adoptar medidas específicas dirigidas a ayudar a ciertas categorías de personas desfavorecidas", por lo que "puede... crear un nuevo contrato de trabajo al objeto de facilitar" la inserción profesional de algún grupo social, como es el caso: Considerandos 16 y 17).

Asimismo, rechaza que esta figura sea contraria al derecho al trabajo. En efecto, "según los recurrentes, la motivación del despido y el carácter contradictorio del procedimiento constituyen garantías de este derecho", por lo que su supresión afectaría al mismo. Sin embargo, a juicio del Consejo, «incumbe al legislador, ...en virtud del artículo 34 de la Constitución..., establecer las reglas propias para asegurar, conforme al... Preámbulo constitucional de 1946, el derecho de cada uno a obtener un empleo, permitiendo el ejercicio de este derecho al mayor número posible y, en su caso, esforzándose para remediar la precariedad". Sin embargo, y dado que —una vez más se recuerda - "el Consejo Constitucional no dispone de un poder general de apreciación y de decisión de la misma naturaleza que el de Parlamento..., no le corresponde investigar si el objetivo que se ha marcado el legislador podría alcanzarse por otras vías, en tanto las modalidades adoptadas por la ley recurrida no son manifiestamente inapropiadas a la finalidad que se persigue" (Considerandos 18 a 21). En cualquier caso, señalaba que "corresponderá al empleador, en caso de recurso, indicar los motivos de la ruptura a fin de permitir al juez que verifique que son lícitos y que sancione un eventual abuso de derechon.

Finalmente, el Juez constitucional desestima también, por diversas razones de fondo y forma, las alegaciones según las cuales el CPE supondría una vio- 
lación del artículo 4 de la Declaración de Derechos de 1789, y de los derechos a la defensa y al recurso, así como de la Carta Social Europea, de una Convención Internacional del Trabajo y de la directiva 2000/78/CE, de 27 de noviembre de 2000.

Asimismo, el Consejo desestima las alegaciones formuladas contra los artículos 48 y 49 (que establecen el "contrato de responsabilidad parental" y la posibilidad de suspensión de ciertas prestaciones familiares, en términos que se consideran suficientemente precisos) y 51 (que permite al alcalde de un municipio ciertas iniciativas respecto a los autores de ciertas contravenciones que causen perjuicio al municipio). Por todo lo cual, la ley se publicó en el Journal Officiel del día 2 de abril, entrando en vigor, sin los artículos declarados inconstitucionales.

Pese a lo cual, el debate no finalizó ahí. En efecto, el día después de hacerse pública la decisión del Consejo, el 31 de marzo, el Presidente Chirac compareció en un discurso televisado en el que anunció su intención de promulgar la ley sobre igualdad de oportunidades, incluyendo las disposiciones relativas al CPE. Sin embargo, hizo pública su intención de solicitar que el Gobierno propusiese al Parlamento una nueva ley modificativa de la anterior, que en particular reduciría de dos años a uno el período de "consolidación", estableciendo igualmente el derecho del trabajador de conocer las causas de su despido. De esta forma, y demostrando una vez más las peculiaridades de la vida política e institucional francesa, anunció que la ley que iba a promulgar no sería aplicada en este respecto, ya que solicitaba al Gobierno que "adoptase todas las disposiciones necesarias para que, en la práctica, no pudiese firmarse ningún contrato que no integrase plenamente estas modificaciones ${ }^{26}$.

Así las cosas, la reacción gubernamental se tradujo en una nueva «ley sobre el acceso de los jóvenes a la vida activa en la empresa", aprobada de manera más que acelerada, en tan sólo cuatro días ${ }^{27}$, y que elimina la discutida figura del CPE.

\section{OTRAS DISPOSICIONES DE INTERÉS}

Para finalizar esta breve crónica, y al margen de que evidentemente otras muchas disposiciones de interés han sido aprobadas durante este período, basta simplemente mencionar que, como también se apuntó al inicio de estas páginas, las revueltas callejeras de octubre-noviembre de 2005 encontraron

26 Datos procedentes de la voz Contrat Première Embauche, en la "wikipedia" (http://fr. wikipedia.org).

27 Loi n. ${ }^{\circ} 2006-457$ du 21 avril 2006 sur l'accès des jeunes à la vie active en entreprise. El texto fue formulado como proposición de ley el 10 de abril: el ponente de la Asamblea depositó su informe el 11 de abril; la Asamblea lo aprobó al día siguiente, fecha en la que también se presentó el informe del ponente del Senado, de forma que la Cámara Alta pudo aprobar el texto, sin modificaciones, el día 13. 
asimismo eco legislativo. Concretamente, debe citarse aquí la Ley 2005-1425, de 18 de noviembre, que prorrogó la aplicación del estado de urgencia previsto por la ley n. ${ }^{\text {s }} 55-385$, de 3 de abril de 1955. Se trataba, en definitiva, de responder a una situación de violencia urbana "dirigida sin distinción contra personas y bienes", para lo cual un Decreto de 8 de noviembre estableció este estado de urgencia durante un plazo de 12 días, que con la ley se prorrogó por tres meses más a partir del día 21 de noviembre, previéndose la posibilidad de su finalización anticipada por virtud de un Decreto del Consejo de Ministros, del que habría de darse cuenta inmediata al Parlamento.

ABSTRACT. The horizon of the double - presidential and legislativeelectoral process to be held in the spring of 2007 has undoubtedly marked the last year of the French political and institutional life, especially in a context in which different crisis have been posed. Thus, some laws have had to deal with the new problems coming from the new electoral calendar, while public opinion has been very sensitive to some initiatives such as the "Contrat Première Embauche" (CPE) that, after having tried to reform the labour market for easing the access of young people, have been hardly debated, contested and, at the end, approved... and not applied. 